\title{
Study of Zernike Polynomials Properties for Oblique Elliptical Aperture at an Angle $(\pi / 4)$ with $\mathrm{X}$-Axis
}

\author{
Sanaa G. Abd ${ }^{1}$, Sundus Y. Hasan ${ }^{2}$ \\ Physics Department, Education College for Girls, Kufa University, Najaf, Iraq
}

\begin{abstract}
In this research, some of the optical properties have been studied for oblique elliptical aperture at an angle ( $\pi / 4)$ with $x$ axis, by using Zernike polynomials. Zernike polynomials for circular aperture and Gram Schmidt orthogonalization method were adopted to find Zernike polynomials for the new aperture. And in this case the equations used are very complex, therefore, new coordinates $m$ and $n$ were used, that they were oblique at $(\pi / 4)$ to both $x$ and $y$ axes respectively. The relationship between Zernike polynomials for the new aperture with first and third order aberrations was derived. And it found that aberrations of high orders were balanced with aberrations of lower orders, for example, third order coma aberration were balanced with first order tilt error, while the third order spherical and astigmatism aberrations were balanced with focus aberration of first order. The standard deviation is also found in this research for balanced and unbalanced aberrations for any value of aspect ratio.
\end{abstract}

Keywords: Zernike polynomials, stander deviation, aberrations, astigmatism

\section{Introduction}

Zernike polynomials considered as one of important topics in optics. So many studies and researches were done from its first study in 1934 by F. Zernike[1], which it bears his name, who used it in testing spherical mirrors, and then by many researchers who extended their studies to apertures other than circular[2-5 ]. This research is interesting in the elliptical aperture, where the circular aperture tilted at some angle becomes an elliptical aperture, and for human eye, which is an optical system of wide field of view. Another examples of elliptical pupil is in shearing interferometry and in imaging and testing fold mirrors[6]. in 2007, V. N. Mahajan studied the Zernike horizontal elliptical aperture, who found the first 15 elliptical Zernike polynomials in Cartizian and polar coordinates[7]. While in 2012 Sundus Y. and Ali S. studied these polynomials for elliptical annular aperture[ 8]. In 2014 also, Jose A. Díaz and R. Navarro studied Orthonormal polynomials for elliptical wavefronts with certain orientations[6 ]. In this research the interesting is on elliptical aperture inclined at an angle $\pi / 4$, to $\mathrm{x}$-axis. Point spread function for this aperture were studied by S. Y. Hasan and W. H. Tarchan in 2014[ 9].

\section{Oblique Elliptical aperture at an angle ( $\pi$ / 4) with $x$-axis}

The current study includes an elliptical aperture tilted at an angle $(\pi / 4)$ to the $\mathrm{x}$-axis, and it is located inside a circle of an area equal $\pi$ or circle of unit radius, (figure 1).

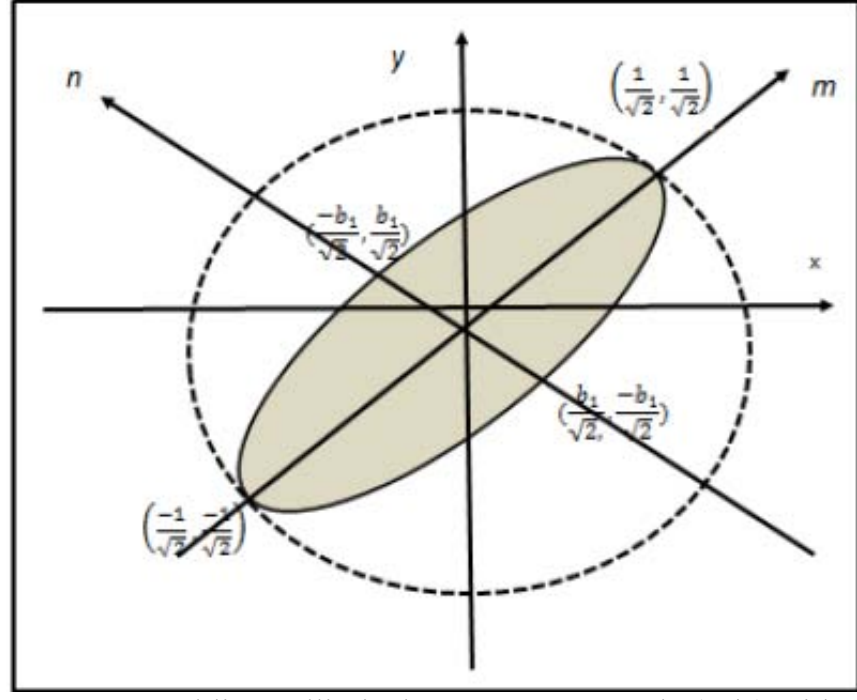

Figure 1: oblique elliptical aperture at anangle $(\pi / 4)$ with the $\mathrm{x}$-axis.

In the next section the Zernike polynomials (ZP) for this aperture would be studied, but the equation will be very difficult to solve, so, in this research the $\mathrm{x}-\mathrm{y}$ coordinates will be changed to $\mathrm{m}-\mathrm{n}$ coordinates which are inclined to original one by $\pi / 4$, as follows:

The equation of $m$ axis is

$$
y=-x \rightarrow y-x=0 \text { so } m=y+x
$$

While the equation for $\mathrm{n}$ axis axis is

$$
y=-x \rightarrow y+x=0 \text { so } n=y-x
$$

The value of $x$ and $y$ in terms of $m$ and $n$ are:

And thus the ellipse equation

$$
\begin{gathered}
m+n=2 y \rightarrow y=\frac{m+n}{2} \\
m-n=2 x \rightarrow x=\frac{m-n}{2}
\end{gathered}
$$

$$
\frac{m^{2}}{a^{2}}+\frac{n^{2}}{b^{2}}=2
$$




\section{International Journal of Science and Research (IJSR) \\ ISSN (Online): 2319-7064 \\ Index Copernicus Value (2013): 6.14 | Impact Factor (2014): 5.611}

Where $\mathrm{a}$ and $\mathrm{b}$ are the semi primary and secondary axes of ellipse. And thus it can be concluded that the limits of ellipse are:

$$
\begin{gathered}
-\sqrt{2}<m<\sqrt{2} \text { and }-b_{1} \sqrt{2-\frac{m^{2}}{2}}<n< \\
b_{1} \sqrt{2-\frac{m^{2}}{2}}, \\
\ldots \ldots .(6)
\end{gathered}
$$

Where from fig.(1), (a) of eq.(5) equals to $\sqrt{2}$, because $\mathrm{m}=1 / \sqrt{2}+1 / \sqrt{2}=\sqrt{2}$.

\section{Zernike polynomials for Oblique Elliptical aperture at an angle $(\pi / 4)$ with $x$-axis}

The Zernike polynomials (ZP) were suggested to describe wave aberration functions over circular pupils of unit radius. Individual terms, of these polynomial are mutually orthogonal over the limits of unit circle andcan be easily normalized to form an orthonormal basis. These polynomials were lost their important properties when the pupils are noncircular. To find ZP for the oblique aperture under interest, Gram Shmidtorthogonalization method was used on the circular Zernike polynomials but on the limits of the new aperture mentioned in the last section, eq. (6). The sequence of circular Zernike polynomials used is those as in reference [10]. Gram Shmidtorthogonalization method can be illustrated as follows: To convert the two circular ZP Z1 and $\mathrm{Z} 2$ to two polynomials orthogonal in the limits of the new aperture [11], the following equation used:

$$
\begin{aligned}
Z_{2}^{\prime}(n, m)= & Z_{2}(n, m)-\frac{\int_{-m}^{m} \int_{-n}^{n} Z_{1}(n, m) Z_{2}(n, m) d n d m}{\int_{-m}^{m} \int_{-n}^{n} Z_{2}(n, m) Z_{2}(n, m) d n d m} \\
& * Z_{1}(n, m) \quad \ldots \ldots .(7)
\end{aligned}
$$

Where $\mathrm{C}$ is normalization constant

dxdy turned into a dmdn using Jacoppin

Thus, the equations (7) and (8) can be written as:

While the equation of normalization that convert the polynomials into normalized polynomials is[11]

$1=C^{2} \frac{\int_{-m}^{m} \int_{-n}^{n}(Z(n, m))^{2} d n d m}{\int_{-m}^{m} \int_{-n}^{n} d n d m}$ dxdy turned into a dmdn using Jacoppin method[12]:

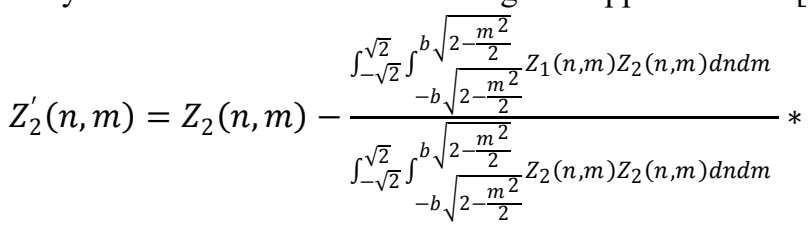$$
Z_{1}(n, m)
$$

method[12]:)

$$
\frac{d m d n}{d x d y}=\left|\begin{array}{ll}
\frac{d m}{d x} & \frac{d m}{d y} \\
\frac{d n}{d x} & \frac{d n}{d y}
\end{array}\right|=\left|\begin{array}{cc}
1 & 1 \\
-1 & 1
\end{array}\right|=2
$$

This means that $d x d y=\frac{1}{2} d m d n$

Thus, the equations (7) and (8) can be written as:

$$
1=C^{2} \frac{\int_{-\sqrt{2}}^{\sqrt{2}} \int^{b} \frac{\sqrt{2-\frac{m^{2}}{2}}}{-b \sqrt{2-\frac{m^{2}}{2}}}(Z(n, m))^{2} d n d m}{\int_{-\sqrt{2}}^{\sqrt{2}} \int_{-b \sqrt{2-\frac{m^{2}}{2}}}^{b \sqrt{2-\frac{m^{2}}{2}}} d n d m}
$$

The last two equations were programed using MATLAB language to find ZP's for the new aperture in $m$ and $n$ coordinates then they transformed again to $\mathrm{x}$ and $\mathrm{y}$ coordinates using equations (1) and (2).

Table (1) represents first fifteen orthogonal polynomials for the new aperture in Cartesian coordinates, while table (2) represents the normalization constants for these polynomials. Table (3) represents these polynomials in polar coordinates.

The MATLAB program, written in this work to get these polynomials, were checked by putting the value of $b=\sqrt{ }$ (where $\sqrt{ }$ is in $m-n$ coordinates which equal to 1 in $x-y$ coordinates) then the circular polynomials is the result. Then these polynomials were drawn in figures (2) in 2-D and 3-D, and the gradient of colors from red to blue shows the amount of convexity and concavity, where the red color refers to the top, while the blue color refers to the bottom, and so on.

Where $\mathrm{C}$ is normalization constant

Table (1): Orthogonal Zernike polynomials for oblique aperture at an angle $(\pi / 4)$ with $\mathrm{x}$-axis in Cartesian coordinates $\left(\rho^{2}=x^{2}+y^{2}\right)$, b is the aspect ratio.

\begin{tabular}{|l|c|}
\hline 1 & 1 \\
\hline 2 & $\mathrm{X}$ \\
\hline 3 & $\mathrm{y}+x\left(\frac{\mathrm{b}^{2}-2}{\mathrm{~b}^{2}+2}(\right.$ \\
\hline 4 & $2 \rho^{2}-\frac{1}{2}\left(1+\frac{b^{2}}{2}\right)$ \\
\hline 5 & $x^{2}-\mathrm{y}^{2}$ \\
\hline 6 & $\frac{3 \rho^{2}\left(\mathrm{~b}^{2}-4\right)+2 \mathrm{xy}\left(3 \mathrm{~b}^{4}-4 \mathrm{~b}^{2}+12\right)+2\left(2 \mathrm{~b}^{2}-\mathrm{b}^{4}\right)}{3 b^{4}-4 b^{2}+12}$ \\
\hline 7 & {$\left[\begin{array}{c}3 \rho^{2} \mathrm{x}-\mathrm{x}\left(\frac{b^{2}}{2}+1\right)+\frac{1}{2} y\left(\frac{b^{2}}{2}-1\right) \\
3 \mathrm{y}^{3}\left(9 \mathrm{~b}^{6}-2 \mathrm{~b}^{4}-4 \mathrm{~b}^{2}+72\right)+ \\
3 \mathrm{x}^{3}\left(9 \mathrm{~b}^{6}-22 \mathrm{~b}^{4}+72\right) \\
+3 y x^{2}\left(9 \mathrm{~b}^{6}-2 \mathrm{~b}^{4}-4 \mathrm{~b}^{2}+72\right)+ \\
3 x \mathrm{y}^{2}\left(9 \mathrm{~b}^{6}-22 \mathrm{~b}^{4}+72\right)+ \\
3 x\left(\frac{3}{4} b^{8}-b^{6}+4 b^{2}+12\right)+3 y\left(\frac{-3}{4} b^{8}-6 b^{6}+4 b^{4}+24 b^{2}+12\right)\end{array}\right]$} \\
\hline
\end{tabular}


International Journal of Science and Research (IJSR)

ISSN (Online): 2319-7064

Index Copernicus Value (2013): 6.14 | Impact Factor (2014): 5.611

\begin{tabular}{|c|c|}
\hline & $/\left[\left(9 b^{6}-2 b^{4}-4 b^{2}+72\right)\right]$ \\
\hline 9 & $6 \rho^{4}-\frac{1}{2} \rho^{2}\left(\frac{b^{2}}{2}+1\right)+3 x y\left(\frac{b^{2}}{2}-1\right)+\frac{3}{32} b^{4}+\frac{b^{2}}{2}+\frac{3}{8}$ \\
\hline 10 & $\begin{array}{c}\left\{\left[-48 b^{6}+448 b^{4}-192 b^{2}\right] x^{3}+\left[-90 b^{8}+168 b^{6}-672 b^{2}+1440\right] x^{2} y+\left[-180 b^{8}+624 b^{6}-1824 b^{4}+2496 b^{2}-2880\right] y^{2} x+\left[24 b^{8}+48 b^{6}+\right.\right. \\
\left.\left.192 b^{2}+144\right] x+\left[84 b^{8}-312 b^{6}+624 b^{4}-672 b^{2}\right] y+\left[-90 b^{8}+168 b^{6}-672 b^{2}+1440\right]\right\} /\left[45 b^{8}+168 b^{6}+568 b^{4}-672 b^{2}\right]\end{array}$ \\
\hline 11 & $\begin{array}{c}\left\{\left[10 b^{6}+36 b^{4}-72 b^{2}-80\right] x^{3}+\left[30 b^{6}-84 b^{4}+168 b^{2}-240\right] x^{2} y+\left[10 b^{6}-36 b^{4}-72 b^{2}+80\right] y^{3}+\left[30 b^{6}-12 b^{4}-24 b^{2}+240\right] y^{2} x+\right. \\
\left.\left[-12 b^{6}+48 b^{2}\right] x+\left[-12 b^{6}+48 b^{4}-48 b^{2}\right] y\right\} /\left[5 b^{6}+6 b^{4}+12 b^{2}+40\right]\end{array}$ \\
\hline 12 & $-4\left(y^{4}-x^{4}\right)+\frac{3}{2} \rho^{2}\left(1-\frac{b 2}{2}\right)$ \\
\hline 13 & $\begin{array}{c}\left\{1 / 4\left[\left(-3840 b^{6}+12032 b^{4}-15360 b^{2}+23040\right) \mathrm{x}^{3} \mathrm{y}+\left(1140 b^{8} \pm 3840 b^{6}+12032 b^{4}-\right.\right.\right. \\
\left.15360 b^{2}+23040\right) \mathrm{y}^{3} \mathrm{x}+\left(1140 b^{8}-1920 b^{6}+7680 b^{4}-23040\right) \mathrm{y}^{2} \mathrm{x}^{2}+720 b^{8}-960 b^{6}+ \\
3840 b^{2}-11520\left(\mathrm{y}^{4}+\mathrm{x}^{4}\right)+\left(-45 b^{10}-750 b^{8}+2328 b^{6} 6000 b^{2}+1140\right) \rho^{2} \\
\left.+\left(-90 b^{10}-120 b^{8}-336 b^{6}-672 b^{4}-3360 b^{2}-2880\right) \mathrm{xy}\right] \\
\left.+30 \mathrm{~b}^{10} / 4-6 \mathrm{~b}^{8}-96 \mathrm{~b}^{4} / 4-120 \mathrm{~b}^{2}\right\} /\left[45 \mathrm{~b}^{8}-120 \mathrm{~b}^{6}+376 \mathrm{~b}^{4}-480 \mathrm{~b}^{2}+720\right] \\
\end{array}$ \\
\hline 14 & $10\left(x^{5}-2 x^{3} y^{2}+y^{4} x\right)-3 x^{3}\left(b^{2}+2\right)-3 b^{2}\left(y^{2} x-y x^{2}\right)+y^{3}\left(b^{2}-2\right)+\left(\frac{9}{3}+\frac{9}{32} b^{2}\right) x+3 y\left(\frac{-b^{4}}{16}-2 x^{2}\right)$ \\
\hline 15 & $\begin{array}{c}\mathrm{y}^{3}\left(\frac{960}{32} \mathrm{~b}^{8}-\frac{3840}{32} \mathrm{~b}^{6}-\frac{15360}{32} \mathrm{~b}^{2}-\frac{15360}{32}\right)+\mathrm{y}\left(\frac{45}{32} \mathrm{~b}^{10}-\frac{60}{32} \mathrm{~b}^{8}-\frac{24}{32} \mathrm{~b}^{6}+76.50 \mathrm{~b}^{2}+45\right)+ \\
\mathrm{x}\left(-\frac{114}{32} \mathrm{~b}^{8}+\frac{1320}{32} \mathrm{~b}^{6}-82.50 \mathrm{~b}^{4}+28.50 \mathrm{~b}^{2}+\frac{76800}{32} \mathrm{y}^{3} \mathrm{x}-45\right)\end{array}$ \\
\hline
\end{tabular}

Table 2: The normalization constants for polynomials of oblique aperture

\begin{tabular}{|c|c|}
\hline $\mathrm{C}_{1}$ & 1 \\
\hline \multirow[t]{2}{*}{$\mathrm{C}_{2}$} & 4 \\
\hline & $\sqrt{\left(b^{2}+2\right)}$ \\
\hline \multirow[t]{2}{*}{$\mathrm{C}_{3}$} & $\sqrt{\left(2 b^{2}+4\right)}$ \\
\hline & $\frac{b}{0}$ \\
\hline \multirow[t]{2}{*}{$\mathrm{C}_{4}$} & $(48)$ \\
\hline & $\sqrt{\left(3 b^{4}-4 b^{2}+12\right)}$ \\
\hline \multirow[t]{2}{*}{$\mathrm{C}_{5}$} & $\sqrt{12}$ \\
\hline & $\overline{\mathrm{b}}$ \\
\hline \multirow[t]{2}{*}{$\mathrm{C}_{6}$} & $\sqrt{\left(9 b^{4}-12 b^{2}+36\right)}$ \\
\hline & $\sqrt{\left(2 b^{2}\right)}$ \\
\hline \multirow[t]{2}{*}{$\mathrm{C}_{7}$} & 32 \\
\hline & $\sqrt{\left(9 b^{6}-2 b^{4}-4 b^{2}+72\right)}$ \\
\hline \multirow[t]{2}{*}{$\mathrm{C}_{8}$} & $1152 b^{6}-256 b^{4}-512 b^{2}+9216$ \\
\hline & $\sqrt{45 b^{10}-168 b^{8}+568 b^{6}-672 b^{4}+720 b^{2}}$ \\
\hline \multirow[t]{2}{*}{$\mathrm{C}_{9}$} & 5120 \\
\hline & $\sqrt{45 b^{8}-120 b^{6}+376 b^{4}-480 b^{2}+720}$ \\
\hline \multirow[t]{2}{*}{$\mathrm{C}_{10}$} & $\longdiv { 4 5 b ^ { 8 } - 1 6 8 b ^ { 6 } + 5 6 8 b ^ { 4 } - 6 7 2 b ^ { 2 } + 7 2 0 }$ \\
\hline & $\sqrt{\frac{5}{4} b^{10}+\frac{3}{2} b^{8}+3 b^{6}+10 b^{4}}$ \\
\hline \multirow[t]{2}{*}{$\mathrm{C}_{11}$} & $\overline{5 b^{6}+6 b^{4}+12 b^{2}+40}$ \\
\hline & $\sqrt{2 b^{6}}$ \\
\hline \multirow[t]{2}{*}{$\mathrm{C}_{12}$} & 4 \\
\hline & $\sqrt{\sqrt{4 b^{6}-15 b^{4}+b^{2}}}$ \\
\hline \multirow[t]{2}{*}{$\mathrm{C}_{13}$} & $1800 b^{8}-4800 b^{6}+15040 b^{4}-19200 b^{2}+28800$ \\
\hline & $\sqrt{35 b^{12}-120 b^{10}+456 b^{8}-480 b^{6}+560 b^{4}}$ \\
\hline \multirow[t]{2}{*}{$\mathrm{C}_{14}$} & 49152 \\
\hline & $\sqrt{75 b^{10}-165 b^{8}-4 b^{6}+732 b^{4}-1040 b^{2}+2400}$ \\
\hline
\end{tabular}

\begin{tabular}{|c|c|}
\hline $\mathrm{C}_{15}$ & $5 b^{6}+11 b^{4}+12 b^{2}+40$ \\
\hline & $\sqrt{\frac{0.085 b^{14}-0.569 b^{12}+2.272 b^{10}+5.234 b^{8}+0.909 b^{6}}{0.911 b^{4}+5.468 b^{2}}}$ \\
\hline
\end{tabular}

Table 3: Orthogonal Zernike polynomials for oblique aperture at an angle $(\pi / 4)$ with $\mathrm{x}$-axis in polar coordinates, $\mathrm{b}$ is the aspect ratio.

\begin{tabular}{|l|l|}
\hline 1 & $\rho^{2}=x^{2}+y^{2}, \quad x=\rho \cos (\alpha), \quad y=\rho \sin (\alpha)$ \\
\hline 2 & $1 / 16[\rho \cos (\alpha)]$ \\
\hline 3 & $1 / 6\left[\rho \sin (\alpha)-\frac{1}{3} \rho \cos (\alpha)\right]$ \\
\hline 4 & $11 / 482\left[\rho^{2}-\frac{3}{4}\right]$ \\
\hline 5 & $1 / 12\left[\rho^{2} \cos (2 \alpha)\right]$ \\
\hline 6 & $2 / 33\left[\rho^{2} \sin (2 \alpha)-\frac{9}{11} \rho^{2}+\frac{2}{11}\right]$ \\
\hline 7 & $75 / 1024\left[\rho \cos (\alpha)\left[3 \rho^{2}-\frac{3}{2}\right]-\frac{1}{4} \rho \sin (\alpha)\right]$ \\
\hline 8 & $493 / 960\left[\rho \cos (\alpha)\left[\frac{-492}{300} \rho^{2}+\frac{171}{300}\right]\right.$ \\
\hline 9 & $541 / 5120\left[6 \rho^{4}-\frac{3}{4} \rho^{2} \sin (2 \alpha)-\frac{9}{2} \rho^{2}+\frac{19}{32}\right]$ \\
\hline 10 & $\begin{array}{l}63 / 1972\left[\rho^{3} \cos { }^{3}(\alpha) \frac{1972}{493}+\rho \cos (\alpha)\left[\frac{-1764}{493} \rho^{2}+\right.\right. \\
72493+\rho \sin \alpha[846493 \rho 2-276493]\end{array}$ \\
\hline 11 & $\left.\left.\begin{array}{c}2 / 63\left[\rho^{3} \sin (3 \alpha)+\frac{5}{63} \rho^{3} \sin (\alpha)+\rho \sin (\alpha)\left[\frac{5}{7} \rho^{2}\right.\right. \\
21\end{array}\right]+\rho \cos (\alpha)\left[\frac{-37}{21} \rho^{2}+\frac{4}{7}\right]\right]$ \\
\hline 13 & $\begin{array}{c}451 / 21640\left[\rho^{2} \sin (2 \alpha)\left[4 \rho^{2}-\frac{3879}{2164}\right]-\frac{1980}{541} \rho^{4}+\right. \\
\left.\cos (2 \alpha)\left[4 \rho^{2}-\frac{9}{4}\right]\right]\end{array}$ \\
\hline
\end{tabular}




\begin{tabular}{|c|c|}
\hline 14 & $\begin{array}{c}651 / 16384\left[\frac{-1}{2} \rho^{3} \sin (3 \alpha)+\rho \sin (\alpha)\left[\frac{-3}{2} \rho^{2}+\frac{9}{16}\right]\right. \\
\left.+\rho \cos (\alpha)\left[10 \rho^{4}-9 \rho^{2}+\frac{57}{32}\right]\right]\end{array}$ \\
\hline 15 & $\begin{aligned} 1365 / 63488\left[\frac{21}{62} \rho^{3} \sin (3 \alpha)+\frac{1}{2} \rho^{3}\right. \\
\quad+\rho \sin (\alpha)\left[10 \rho^{4}-\frac{495}{62} \rho^{2}\right. \\
\left.\quad+\frac{1389}{992}\right] \\
+\rho \cos (\alpha)\left[\frac{210}{31} \rho^{4}+\frac{285}{62} \rho^{2}\right. \\
\left.\left.\quad+\frac{639}{992}\right]\right]\end{aligned}$ \\
\hline 16 & $\begin{array}{c}7207 / 114688\left[\rho^{3} \sin (2 \alpha)\left[-5 \rho^{2}+\frac{9}{4}\right]+20 \rho^{6}\right. \\
\left.-\frac{45}{2} \rho^{4}+\frac{57}{8} \rho^{2}-\frac{63}{128}\right]\end{array}$ \\
\hline 17 & $\begin{array}{c}32 / 2255\left[\rho^{4} \cos (4 \alpha)+\rho^{2} \sin (2 \alpha)\left[\frac{1380}{451} \rho^{2}-\frac{504}{451}\right]\right. \\
\left.\left.-\frac{75}{41} \rho^{4}+\frac{360}{451} \rho^{2}-\frac{24}{451}\right]\right]\end{array}$ \\
\hline 18 & $1 / 65\left[\rho^{4} \sin (4 \alpha)+\rho^{2} \cos (2 \alpha)\left[\frac{-30}{13} \rho^{2}+\frac{12}{13}\right]\right]$ \\
\hline 19 & $\begin{aligned} 2801 / 174720\left[\rho^{3}\right. & \sin (\alpha) \cos ^{2}(\alpha) \frac{2058}{2730} \\
& +\frac{1}{2730} \rho^{3} \cos ^{3}(\alpha)\left[54600 \rho^{2}\right. \\
& -27772] \\
& +\frac{1}{2730} \rho \cos (\alpha)\left[-51240 \rho^{4}\right. \\
& \left.+26340 \rho^{2}-558\right] \\
& +\frac{1}{2730} \rho \sin (\alpha)\left[24940 \rho^{4}\right. \\
& \left.\left.-17322 \rho^{2}+2328\right]\right]\end{aligned}$ \\
\hline 20 & $\begin{array}{l}4843 / 313712\left[-20 \rho^{4} \cos ^{2}(\alpha) \sin (\alpha)\right. \\
+10.0278 \rho^{2} \cos ^{2}(\alpha) \sin (\alpha) \\
-0.5272 \rho^{4} \sin (\alpha)+0.6997 \rho^{2} \sin (\alpha) \\
-0.3685 \sin (\alpha)-3.8486 \rho^{4} \cos ^{3}(\alpha) \\
+12.0374 \rho^{2} \cos ^{3}(\alpha)+12.4732 \rho^{4} \cos (\alpha) \\
\left.-7.4485 \rho^{2} \cos (\alpha)+8.9208 \cos (\alpha)\right]\end{array}$ \\
\hline 21 & $\begin{array}{c}1247 / 57344\left[\frac{-5}{8} \rho^{4} \sin (4 \alpha)+\rho^{2} \cos (2 \alpha)\left[15 \rho^{4}\right.\right. \\
\left.-15 \rho^{2}+\frac{111}{32}\right]\end{array}$ \\
\hline
\end{tabular}

\section{Relationship between Zernike Polynomials and Third-Order Aberrations}

The expansion of wavefront aberration in terms of field independent wavefront can be written as

$W(x, y)=W_{11} \rho \cos \theta+W_{20} \rho^{2}+W_{40} \rho^{4}+W_{31} \rho^{3} \cos \theta+$ $W_{22} \rho^{2} \cos ^{2} \theta$

Because there is no field dependence in these terms, they are not true Seidel aberrations. Wavefront measurement using an interferometer provides only data at a single field point. This causes field curvature to look like focus, and distortion to look like tilt. Therefore, a number of field points must be measured to determine the Seidel aberrations[13] . By using the first nine oblique elliptical Zernike polynomials, the relationship between Zernike polynomials and third-order aberrations can obtained, as follows:

$$
\begin{gathered}
W(x, y)=E_{0}+E_{1} x+E_{2}\left(y+\alpha_{1} x\right)+E_{3}\left(2 \rho^{2}-\alpha_{2}\right) \\
+E_{4}\left(x^{2}-y^{2}\right) \\
+E_{5}\left(2 x y+3 \rho^{2} \frac{\alpha_{3}}{\alpha_{4}}+\frac{\alpha_{5}}{\alpha_{4}}\right) \\
+E_{6}\left(3\left(x^{3}+y^{2} x\right)-x \alpha_{6}+\frac{1}{2} y \alpha_{7}\right) \\
+E_{7}\left(3\left(y^{3}+y^{2}\right)+3\left(x^{3}+x^{2}\right) \frac{\alpha_{9}}{\alpha_{8}}+3 x \frac{\alpha_{10}}{\alpha_{8}}+3 y \frac{\alpha_{11}}{\alpha_{8}}\right) \\
+E_{8}\left(6 \rho^{4}+\rho^{2} \alpha_{13}+3 y x \alpha_{7+} \alpha_{12}\right) \ldots . .
\end{gathered}
$$

Here E0 to E8 represent weighted constants of linear sum, and

\begin{tabular}{|c|c|}
\hline$\alpha_{1}$ & $\frac{b^{2}-2}{b^{2}+2}$ \\
\hline$\alpha_{2}$ & $\frac{1}{2}\left(1+\frac{b^{2}}{2}\right)$ \\
\hline$\alpha_{3}$ & $\left(\mathrm{~b}^{2}-4\right)$ \\
\hline$\alpha_{4}$ & $\left(3 \mathrm{~b}^{4}-4 \mathrm{~b}^{2}+12\right)$ \\
\hline$\alpha_{5}$ & $\frac{2\left(2 \mathrm{~b}^{2}-\mathrm{b}^{4}\right)}{\alpha^{2}}+1$ \\
\hline$\alpha_{6}$ & $\frac{b^{2}}{2}-1$ \\
\hline$\alpha_{7}$ & $9 \mathrm{~b}^{6}-2 \mathrm{~b}^{4}-4 \mathrm{~b}^{2}+72$ \\
\hline$\alpha_{8}$ & $9 \mathrm{~b}^{6}-22 \mathrm{~b}^{4}+72$ \\
\hline$\alpha_{9}$ & $\frac{3}{4} b^{8}-b^{6}+4 b^{2}+12$ \\
\hline$\alpha_{10}$ & $\frac{-3}{4} b^{8}-6 b^{6}-12$ \\
\hline$\alpha_{11}$ & $\frac{3}{32} b^{4}+\frac{b^{2}}{2}+\frac{3}{8}$ \\
\hline$\alpha_{12}$ & $\frac{3}{2} b^{2}-3$ \\
\hline$\alpha_{13}$ & \\
\hline
\end{tabular}

Figure

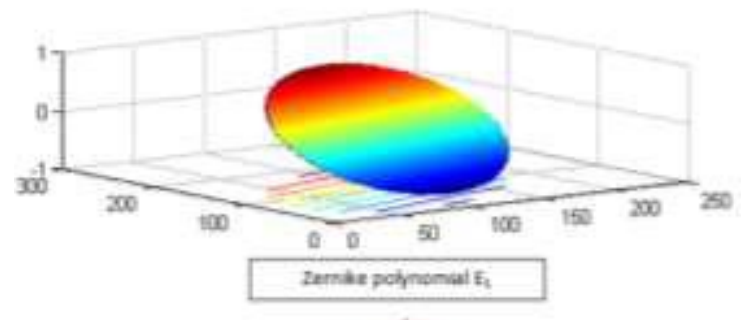




\section{International Journal of Science and Research (IJSR) \\ ISSN (Online): 2319-7064}

Index Copernicus Value (2013): 6.14 | Impact Factor (2014): 5.611
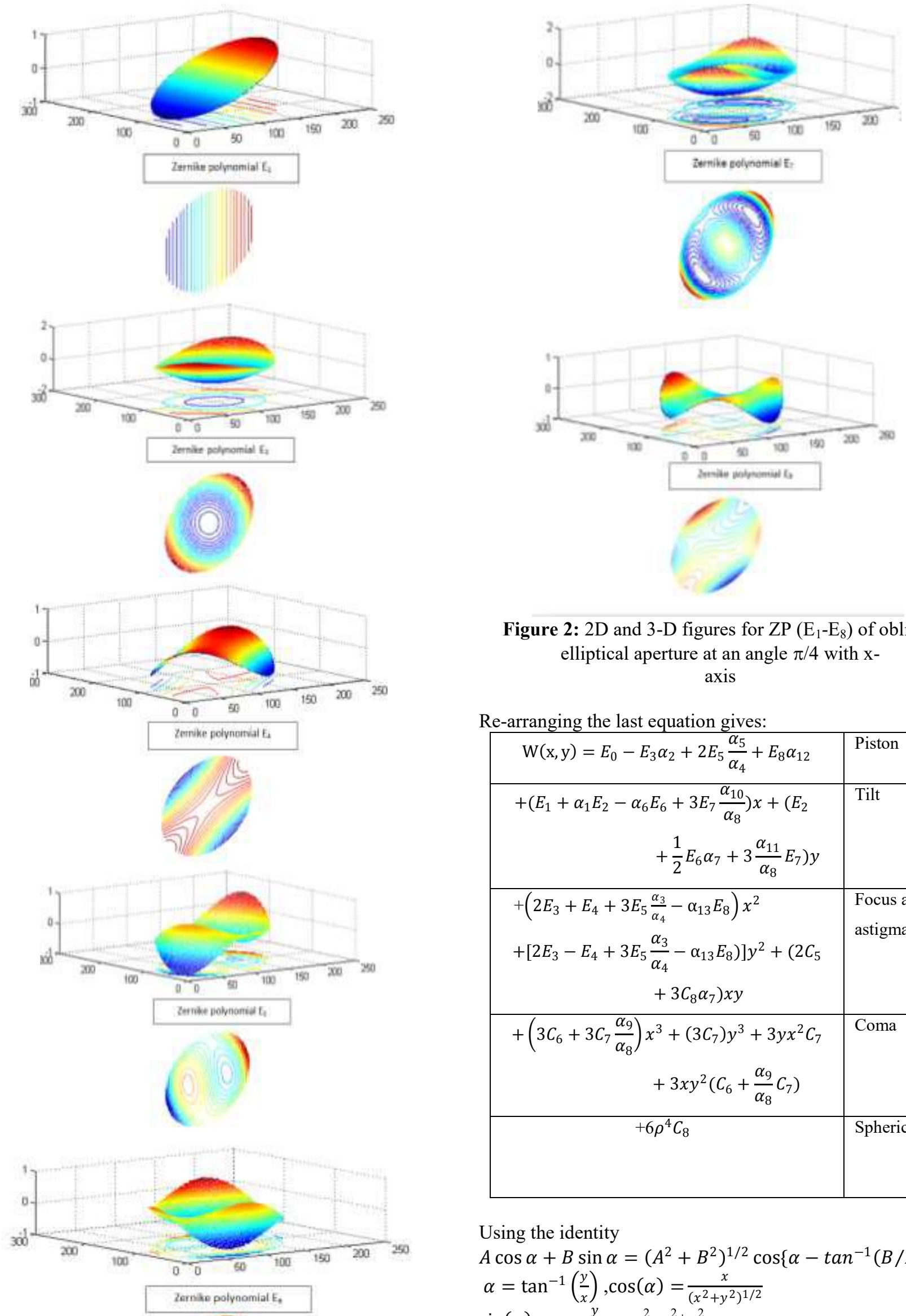

Figure 2: $2 \mathrm{D}$ and 3-D figures for $\mathrm{ZP}\left(\mathrm{E}_{1}-\mathrm{E}_{8}\right)$ of oblique elliptical aperture at an angle $\pi / 4$ with $\mathrm{X}$ axis

Re-arranging the last equation gives:

\begin{tabular}{|c|l|}
\hline $\mathrm{W}(\mathrm{x}, \mathrm{y})=E_{0}-E_{3} \alpha_{2}+2 E_{5} \frac{\alpha_{5}}{\alpha_{4}}+E_{8} \alpha_{12}$ & Piston \\
\hline$+\left(E_{1}+\alpha_{1} E_{2}-\alpha_{6} E_{6}+3 E_{7} \frac{\alpha_{10}}{\alpha_{8}}\right) x+\left(E_{2}\right.$ & Tilt \\
$\left.+\frac{1}{2} E_{6} \alpha_{7}+3 \frac{\alpha_{11}}{\alpha_{8}} E_{7}\right) y$ & \\
\hline$+\left(2 E_{3}+E_{4}+3 E_{5} \frac{\alpha_{3}}{\alpha_{4}}-\alpha_{13} E_{8}\right) x^{2}$ & Focus and \\
$\left.+\left[2 E_{3}-E_{4}+3 E_{5} \frac{\alpha_{3}}{\alpha_{4}}-\alpha_{13} E_{8}\right)\right] y^{2}+\left(2 C_{5}\right.$ & astigmatism \\
$\left.+3 C_{8} \alpha_{7}\right) x y$ & \\
$+\left(3 C_{6}+3 C_{7} \frac{\alpha_{9}}{\alpha_{8}}\right) x^{3}+\left(3 C_{7}\right) y^{3}+3 y x^{2} C_{7}$ & Coma \\
$+3 x y^{2}\left(C_{6}+\frac{\alpha_{9}}{\alpha_{8}} C_{7}\right)$ & \\
\hline$+6 \rho^{4} C_{8}$ & Spherical \\
\hline
\end{tabular}

Using the identity

$A \cos \alpha+B \sin \alpha=\left(A^{2}+B^{2}\right)^{1 / 2} \cos \left\{\alpha-\tan ^{-1}(B / A)\right\}$

$\alpha=\tan ^{-1}\left(\frac{y}{x}\right), \cos (\alpha)=\frac{x}{\left(x^{2}+y^{2}\right)^{1 / 2}}$

$\sin (\alpha)=\frac{y}{\left(x^{2}+y^{2}\right)^{1 / 2}} \rho^{2}=x^{2}+y^{2}$

The term of tilt becomes $\left(E_{1}+\alpha_{1} E_{2}-\alpha_{6} E_{6}+\right.$ $\left.3 E_{7} \frac{\alpha_{10}}{\alpha_{8}}\right) \rho \cos \alpha+\left(E_{2}+\frac{1}{2} E_{6} \alpha_{7}+3 \frac{\alpha_{11}}{\alpha_{8}} E_{7}\right) \rho \sin \alpha=$

Volume 5 Issue 2, February 2016

www.ijsr.net 


\section{International Journal of Science and Research (IJSR) \\ ISSN (Online): 2319-7064}

Index Copernicus Value (2013): 6.14 | Impact Factor (2014): 5.611

$$
\begin{aligned}
& \rho^{2}\left[\left(\alpha_{1} E_{2}-\alpha_{6} E_{6}+3 E_{7} \frac{\alpha_{10}}{\alpha_{8}}\right)^{2}+\left(E_{2}+\frac{1}{2} E_{6} \alpha_{7}+\right.\right. \\
& \left.\left.3 \frac{\alpha_{11}}{\alpha_{8}} E_{7}\right)^{2}\right]^{\frac{1}{2}} \\
& \times \cos \left[\alpha-\tan ^{-1}\left(\frac{\left(E_{2}+\frac{1}{2} E_{6} \alpha_{7}+3 \frac{\alpha_{11}}{\alpha_{8}} E_{7}\right)}{\left(\alpha_{1} E_{2}-\alpha_{6} E_{6}+3 E_{7} \frac{\alpha_{10}}{\alpha_{8}}\right)}\right)\right]
\end{aligned}
$$

As well as,the term of( focus and astigmatism) becomes $\left(2 E_{3}+E_{4}+3 E_{5} \frac{\alpha_{3}}{\alpha_{4}}-\alpha_{13} E_{8}\right) x^{2}+\left[2 E_{3}-E_{4}+3 E_{5} \frac{\alpha_{3}}{\alpha_{4}}-\right.$ $\left.\left.\alpha_{13} E_{8}\right)\right] y^{2}+\left(2 E_{5}+3 E_{8} \alpha_{7}\right) x y$

Written as follows:

And also:

$$
\begin{aligned}
& \left(2 E_{3}+3 E_{5} \frac{\alpha_{3}}{\alpha_{4}}-\alpha_{13} E_{8}\right) \rho^{2}+E_{4}\left(x^{2}-y^{2}\right) \\
& +\left(2 E_{5}+3 E_{8} \alpha_{7}\right) x y \ldots \ldots .
\end{aligned}
$$

$E_{4}\left(x^{2}-y^{2}\right)+\left(2 E_{5}+3 E_{8} \alpha_{7}\right) x y=E_{4}\left[\left(\rho^{2} \cos ^{2}(\alpha)-\right.\right.$

$$
\left.\left.\rho^{2} \sin ^{2}(\alpha)\right)\right]+\left(2 E_{5}+3 E_{8} \alpha_{7}\right) \rho^{2} \cos (\alpha) \sin (\alpha)
$$$$
=E_{4} \rho^{2} \cos (2 \alpha)+\left[\left(2 E_{5}+3 E_{8} \alpha_{7}\right)\right] \frac{1}{2} \rho^{2} \sin (2 \alpha)
$$

$$
\begin{gathered}
=\rho^{2}\left[\left(E_{4}\right)^{2}+\left(\left(\frac{1}{2}\left(2 E_{5}+3 E_{8} \alpha_{7}\right)\right)^{2}\right]^{\frac{1}{2}} \cos [2 \alpha-\right. \\
\tan ^{-1}\left(\frac{\frac{1}{2}\left(2 E_{5}+3 E_{8} \alpha_{7}\right)}{E_{4}}\right) \ldots \ldots \ldots \ldots \ldots \ldots \ldots
\end{gathered}
$$

Since

$$
\cos (2)=\cos ^{2}(\alpha)-\sin ^{2}(\alpha)
$$

$\rho^{2} \cos \left[2\left(\alpha-\tan ^{-1} \frac{2}{4 E_{4}}\right)\right.$

$=\cos ^{2}\left[\alpha-\frac{1}{2} \tan ^{-1}\left(\frac{2 E_{5} \alpha_{3}+3 E_{8} \alpha_{7}}{2 E_{4}}\right)\right]-\sin ^{2}[\alpha-$

$12 \tan -1(2 E 5 \alpha 3+3 E 8 \alpha 72 E 4)$.

Using matched:

$\cos ^{2} \theta-\sin ^{2} \theta=\cos ^{2} \theta-\left(1-\cos ^{2} \theta\right)=\left(-1+2 \cos ^{2} \theta\right)$

$\mathrm{Be}$ the result of the previous equation is:

$=\left[-1+2 \cos ^{2}\left[\alpha-\frac{1}{2} \tan ^{-1}\left(\frac{2 E_{5}+3 E_{8} \alpha_{7}}{2 E_{4}}\right)\right]\right]$

since equation (16) form:

$\rho^{2}\left\{\left[\left(2 E_{3}+3 E_{5} \frac{\alpha_{3}}{\alpha_{4}}\right)-\alpha_{13} E_{8}\right]-\left[\left(E_{4}\right)^{2}+\left(\alpha_{13} E_{8}\right)^{2}\right]\right\}+$

$2 \rho^{2} \cos ^{2}\left[\alpha-\frac{1}{2} \tan ^{-1}\left(\frac{2 E_{5}+3 E_{8} \alpha_{7}}{2 E_{4}}\right)\right]$

And also, the term of coma becones

$$
\begin{aligned}
& {\left[3 \rho^{3} \cos ^{3}(\alpha)\left(E_{6}+E_{7} \frac{\alpha_{9}}{\alpha_{8}}\right)+\left(3 E_{7} \rho^{3} \sin ^{3}(\alpha)\right)\right.} \\
& +3 E_{7} \rho^{3} \sin (\alpha) \cos ^{2}(\alpha) \\
& \left.+\left(E_{6}+\frac{\alpha_{9}}{\alpha_{8}} E_{7}\right) 3 \rho^{3} \cos (\alpha) \sin ^{2}(\alpha)\right] \\
& =\left(E_{6}+\frac{\alpha_{9}}{\alpha_{8}} E_{7}\right) 3 \rho^{3} \cos (\alpha)+3 E_{7} \rho^{3} \sin (\alpha) \text {. } \\
& \operatorname{As}\left(\sin ^{2}(\alpha)+\cos ^{2}(\alpha)\right)=1 \\
& \text { We get } \\
& 3 \rho^{3} \cos (\alpha)\left(E_{6}+E_{7} \frac{\alpha_{9}}{\alpha_{8}}\right) \\
& +3 \rho^{3} E_{7} \sin (\alpha)\left(\sin ^{2}(\alpha)+\cos ^{2}(\alpha)\right) \\
& =3 \rho^{3}\left[\left(E_{6}+E_{7} \frac{\alpha_{9}}{\alpha_{8}}\right)^{2}+\left(E_{7}\right)^{2}\right]^{\frac{1}{2}} \cos \left[\alpha-\tan ^{-1}\left(\frac{E_{7}}{\left(E_{6}+E_{7} \frac{\alpha_{9}}{\alpha_{8}}\right)}\right)\right.
\end{aligned}
$$

Finally, the terms of wavefront aberration, can be written as $W(x, y)=$

$$
\begin{aligned}
& E_{0}-E_{3} \alpha_{2}+2 E_{5} \frac{\alpha_{5}}{\alpha_{4}}+E_{8} \alpha_{12} \\
& \rho^{\frac{1}{2}}\left[\left(\alpha_{1} E_{2}-\alpha_{6} E_{6}+3 E_{7} \frac{\alpha_{10}}{\alpha_{8}}\right)^{2}+\left(E_{2}\right.\right. \\
& +\frac{1}{2} E_{6} \alpha_{7} \\
& \left.\left.+3 \frac{\alpha_{11}}{\alpha_{8}} E_{7}\right)^{2}\right]^{\frac{1}{2}}
\end{aligned}
$$

Piston

$\times \cos [\alpha$$$
\left.-\tan ^{-1}\left(\frac{\left(E_{2}+\frac{1}{2} E_{6} \alpha_{7}+3 \frac{\alpha_{11}}{\alpha_{8}} E_{7}\right)}{\left(\alpha_{1} E_{2}-\alpha_{6} E_{6}+3 E_{7} \frac{\alpha_{10}}{\alpha_{8}}\right)}\right)\right]
$$$$
+\left(\rho^{2}\right)\left\{\left[\left(2 E_{3}+3 E_{5} \frac{\alpha_{3}}{\alpha_{4}}\right)-\alpha_{13} E_{8}\right]-1\right\}
$$

Focus

$$
\begin{aligned}
& \pm \rho^{2}\left[\left(E_{4}\right)^{2} \pm\left(E_{5} \alpha_{3}+\frac{3}{2} E_{8} \alpha_{7}\right)^{2}\right]^{1 / 2} \\
& \times 2 \cos ^{2}\left(\alpha-\frac{1}{2} \tan ^{-1}\left(\frac{2 E_{5}+3 E_{8} \alpha_{7}}{2 E_{4}}\right)\right) \\
& +3 \rho^{3}\left[\left(E_{6}+\frac{\alpha_{9}}{\alpha_{8}} E_{7}\right)^{2}+\left(E_{7} \sin \alpha\right)^{2}\right]^{1 / 2} \\
& \times \cos \left[\alpha-\tan ^{-1} \frac{E_{7}}{\left(E_{6}+E_{7} \frac{\alpha_{9}}{\alpha_{8}}\right)}\right]
\end{aligned}
$$

Astig

matis

Coma

$$
+6 \rho^{4} E_{8}
$$

Spheric

al

The magnitude, sign, and angle of these field-independent aberration terms are listedin Table(4). Note focus take a sign that minimize thecoefficient magnitude, whileastigmatism take a sign opposite to that of focus. Table (4): First and third order aberration in terms of oblique ellipse Zernike coefficients at angle $\pi / 4$ with $\mathrm{x}$ - axis.

\section{Standard Deviation of Balanced and Unbalanced primary Aberration}

The Variation to aberration function given by the following equation:[13]

$$
\sigma^{2}=<W^{2}(n, m)>-<W(n, m)>^{2}
$$

Where the average value of the square of aberration given by (mean square value)

$$
\left\langle W^{2}(n, m)\right\rangle=\frac{\int_{-\sqrt{2}}^{\sqrt{2}} \int^{b} \sqrt{2-\frac{m^{2}}{2}} W^{2}(\mathrm{n}, \mathrm{m}) d n d m}{-\frac{m^{2}}{2}} .
$$

while average value of the aberration function (mean value) aberration writes [13]: 


\section{International Journal of Science and Research (IJSR) \\ ISSN (Online): 2319-7064}

Index Copernicus Value (2013): 6.14 | Impact Factor (2014): 5.611

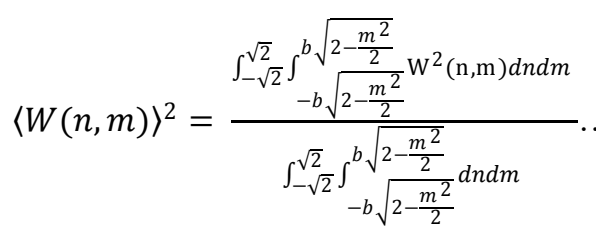

Because orthogonality property, the results of above two equations are:

Then the variance would be:

$$
\begin{array}{r}
\sigma^{2}=<W^{2}(n, m)>-<W(n, m)>^{2}=C_{1}{ }^{2}+C_{2}{ }^{2}+C_{3}{ }^{2}+ \\
C_{4}{ }^{2} \ldots \ldots \ldots
\end{array}
$$

That's mean, that expressing the variance in terms of ZP's makes each coefficient $C_{n}$ represents the standard deviation of the corresponding aberration.

If the aberration function is the value of:

$W=C_{0} E_{0}+C_{1} E_{1}+C_{2} E_{2}+\cdots$

Then the Variance is

$$
\sigma^{2}=\sum_{1}^{n} C_{n}^{2}
$$

Thus, it can be conclude that the variation of aberration is given simply by summing the squares of the normalization functions except the first term or piston $\mathrm{C}_{0}$. The square root of the variance $\left(\sigma^{2}\right)$ represents the standard deviation $(\sigma)$ of aberration.

Now, the standard deviation of balanced and unbalanced aberrations will found:

1) The tilt error on the $x$-axis is the second term of ZP's If the aberration function is the value of:

$W=C_{0} E_{0}+C_{1} E_{1}+C_{2} E_{2}+\cdots$

Then the Variance is

$$
\sigma^{2}=\sum_{1}^{n} C_{n}^{2} \cdots
$$

Thus, it can be conclude that the variation of aberration is given simply by summing the squares of the normalization functions except the first term or piston $\mathrm{C}_{0}$. The square root of the variance $\left(\sigma^{2}\right)$ represents the standard deviation $(\sigma)$ of aberration.

Now, the standard deviation of balanced and unbalanced aberrations will found:

1) The tilt error on the $x$-axis is the second term of ZP's

$$
\begin{aligned}
& E_{2}=\frac{4}{\sqrt{b^{2}+2}} x \\
& x=\frac{\sqrt{b^{2}+2}}{4} E_{2}
\end{aligned}
$$

Applying the equation (27) to obtaine the value of the standard deviation of the tilt aberration on $\mathrm{x}$-axis

$$
\sigma_{\text {tilt }}=\frac{\sqrt{b^{2}+2}}{4}
$$

2) Focus error is found in the fourth term of P's
$E_{4}=\frac{\sqrt{48}}{\sqrt{3 b^{4}-4 b^{2}+12}}\left[2 \rho^{2}-\frac{1}{2}\left(1-\frac{b^{2}}{2}\right)\right]=\frac{2 \sqrt{48}}{\sqrt{3 b^{4}-4 b^{2}+12}}\left[\rho^{2}-\right.$

$\left.\frac{1}{4}\left(1-\frac{b^{2}}{2}\right)\right]$

$\sigma_{f}=\frac{\sqrt{3 b^{4}-4 b^{2}+12}}{2 \sqrt{48}}$

3) Fifth polynomial represents astigmatism aberration:

$E_{5}=\frac{\sqrt{12}}{b}\left[x^{2}-y^{2}\right]$

$E_{5}=\frac{\sqrt{12}}{b}\left[2 x^{2}-\rho^{2}\right] \rightarrow x^{2}-\frac{\rho^{2}}{2}=\frac{b^{2}}{2 \sqrt{12}} E_{5}$

Thus, the standard deviation of the balanced astigmatism

$\sigma_{b a}=\frac{b}{2 \sqrt{12}}$

It's obvious astigmatism balanced with focus error of the first order.

To determine $\sigma$ to un-balanced standard divination (Seidel), it must be written as

$x^{2}=\frac{b E_{5}}{2 \sqrt{12}}+\frac{\rho^{2}}{2}$

And substitude the value of $\rho^{2}$, getting:

$$
\begin{aligned}
& x^{2}=\frac{b E_{5}}{2 \sqrt{12}}+\left[\frac{\sqrt{3 b^{4}-4 b^{2}+12}}{4 \sqrt{48}} E_{4}+\frac{1}{8}\left(1-\frac{b^{2}}{2}\right)\right] \\
\sigma_{a}= & \sqrt{\frac{b^{2}}{48}+\frac{\left(3 b^{4}-4 b^{2}+12\right)}{768}}
\end{aligned}
$$

4)Coma aberration is found in the seventh polynomial:

$$
\begin{gathered}
E_{7}=\frac{32}{\sqrt{9 b^{6}-2 b^{4}-4 b^{2}+72}}\left[3 \rho^{2} x+x\left(\frac{b^{2}}{2}+1\right)\right. \\
\left.+\frac{1}{2} y\left(\frac{b^{2}}{2}-1\right)\right] \\
E_{7}=\frac{96}{\sqrt{9 b^{6}-2 b^{4}-4 b^{2}+72}}\left[\rho^{2} x+\frac{x}{3}\left(\frac{b^{2}}{2}+1\right)\right. \\
\left.+\frac{1}{6} y\left(\frac{b^{2}}{2}-1\right)\right]
\end{gathered}
$$

Thus, the balanced standard deviation of the aberration:

$\sigma_{b c}=\frac{\sqrt{9 b^{6}-2 b^{4}-4 b^{2}+72}}{96}$

So, comma aberration is balanced with tilt error in $\mathrm{x}$ and $\mathrm{y}$ coordinates, and to find $\sigma$ Seidel aberration of comma, it should be written as:

$$
\begin{aligned}
\frac{96}{\sqrt{9 b^{6}-2 b^{4}-4 b^{2}+72}} & E_{7} \\
& =\left[\rho^{3} \cos (\alpha)-\frac{x}{3}\left(\frac{b^{2}}{2}+1\right)\right. \\
& \left.+\frac{1}{6} y\left(\frac{b^{2}}{2}-1\right)\right]
\end{aligned}
$$

Where $x=\frac{\sqrt{b^{2}+2}}{4} E_{2}$ and $\mathrm{y}=\frac{b}{\sqrt{2 b^{2}+4}} E_{3}+\frac{\sqrt{b^{2}+2}}{4} E_{2}\left(\frac{b^{2}-2}{b^{2}+2}\right)$ So: 


$$
\begin{aligned}
& \rho^{3} \cos (\alpha)=\frac{\sqrt{9 b^{6}-2 b^{4}-4 b^{2}+72}}{96} E_{7} \\
&+\frac{\sqrt{b^{2}+2}}{12}\left(\frac{b^{2}}{2}+1\right) E_{2} \\
&-\frac{1}{6}\left(\frac{b^{2}}{2}-1\right)\left[\frac{b}{\sqrt{2 b^{2}+4}} E_{3}\right. \\
&\left.-\frac{\sqrt{b^{2}+2}}{4} E_{2}\left(\frac{b^{2}-2}{b^{2}+2}\right)\right] \\
& \rho^{3} \cos (\alpha)=\frac{\sqrt{9 b^{6}-2 b^{4}-4 b^{2}+72}}{96} E_{7} \\
&-\frac{1}{6}\left(\frac{b^{2}}{2}-1\right) \frac{b}{\sqrt{2 b^{2}+4}} E_{3} \\
&+\frac{\sqrt{\left(b^{2}+2\right)}}{12} E_{2}\left[\frac{1}{6}\left(\frac{b^{2}}{2}-1\right) \frac{1}{12}\left(\frac{b^{2}-2}{b^{2}+2}\right)-\left(\frac{b^{2}}{2}+1\right)\right]
\end{aligned}
$$

By applying equation of standard diviation:

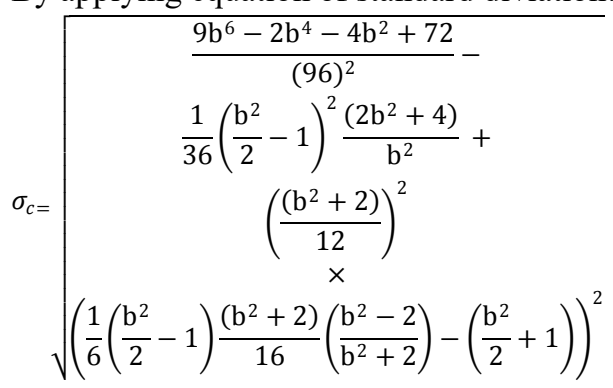

5) Spherical aberration is found in the ninth polynomial

$$
\begin{gathered}
E_{9}=\sqrt{\frac{4 \times 1280}{45 b^{8}-120 b^{6}+376 b^{4}-480 b^{2}+720}}\left(6 \rho^{4}\right. \\
-\frac{1}{2} \rho^{2}\left(\frac{b^{2}}{2}-1\right)+3 x y\left(\frac{b^{2}}{2}-1\right) \\
\left.+\frac{3}{32} b^{4}+\frac{b^{2}}{2}+\frac{3}{8}\right)
\end{gathered}
$$

Therefore, the standard deviation of the balanced spherical aberration is:

$$
\begin{gathered}
E_{9}=\sqrt{\frac{184320}{45 b^{8}-120 b^{6}+376 b^{4}-480 b^{2}+720}}\left(\rho^{4}\right. \\
-\frac{1}{2} \rho^{2}\left(\frac{b^{2}}{2}-1\right) \\
\left.\quad+\frac{1}{2} x y\left(\frac{b^{2}}{2}-1\right)+\frac{1}{64} b^{4}+\frac{b^{2}}{12}+\frac{3}{48}\right) \\
\sigma_{b s}=\sqrt{\frac{45 b^{8}-120 b^{6}+376 b^{4}-480 b^{2}+720}{184320}}
\end{gathered}
$$

Spherical aberration is balanced with focus error and astigmatism, and to determine the $\sigma$ of the Seidel spherical aberration,

$$
\begin{aligned}
\rho^{4}=\sqrt{\frac{45 b^{8}-120 b^{6}+376 b^{4}-480 b^{2}+720}{184320}} E_{9} \\
+\frac{1}{2} \rho^{2}\left(\frac{b^{2}}{2}+1\right)-\frac{1}{2} x y\left(\frac{b^{2}}{2}-1\right)-\frac{b^{4}}{64} \\
-\frac{b^{2}}{12}-\frac{3}{48}
\end{aligned}
$$

where

$$
\begin{aligned}
& \rho^{2}=\frac{\sqrt{3 b^{4}-4 b^{2}+12}}{2 \sqrt{48}} E_{4}+\frac{1}{4}\left(1-\frac{b^{2}}{2}\right) \text { and } x y= \\
& {\left[\frac{\sqrt{2} b^{2}}{2 \sqrt{9 b^{4}-12 b^{2}+36}} E_{6}+\frac{9}{22} \rho^{2}-\frac{2}{22}\right]} \\
& \rho^{4}=\sqrt{\frac{45 b^{8}-120 b^{6}+376 b^{4}-480 b^{2}+720}{184320}} E_{9} \\
& +\frac{1}{2}\left[\frac{\sqrt{3 b^{4}-4 b^{2}+12}}{2 \sqrt{48}} E_{4}\right]\left(\frac{b^{2}}{2}+1\right) \\
& -\frac{1}{2}\left[\left[\frac{\sqrt{2} b^{2}}{2 \sqrt{9 b^{4}-12 b^{2}+36}} E_{6}\right.\right. \\
& \left.\left.+\frac{9}{22}\left[\frac{\sqrt{3 b^{4}-4 b^{2}+12}}{2 \sqrt{48}} E_{4}\right]-\frac{2}{22}\right]\right]\left(\frac{b^{2}}{2}\right. \\
& -1)-\frac{b^{4}}{64}+\frac{b^{2}}{12}+\frac{3}{48} \\
& \rho^{4}=\sqrt{\frac{45 b^{8}-120 b^{6}+376 b^{4}-480 b^{2}+720}{184320}} E_{9} \\
& -\frac{1}{2} \frac{\sqrt{2} b^{2}}{2 \sqrt{9 b^{4}-12 b^{2}+36}} E_{6}\left(\frac{b^{2}}{2}-1\right) \\
& +\frac{1}{2} \frac{\sqrt{3 b^{4}-4 b^{2}+12}}{2 \sqrt{48}} E_{4}\left[\left(\frac{b^{2}}{2}+1\right)\right. \\
& \left.-\frac{9}{22}\left(\frac{b^{2}}{2}-1\right)\right]
\end{aligned}
$$

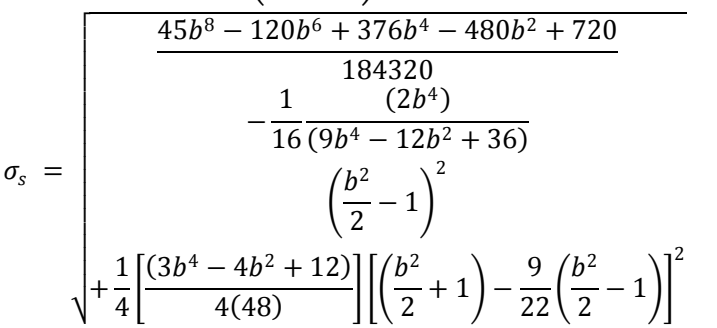

Table (5) represents the standard deviation for balanced and un-balanced aberrations of oblique aperture with angle $\pi / 2$ to $\mathrm{x}$-axis and compared with those values of circular aperture of reference [7]. 


\begin{tabular}{|c|c|c|}
\hline Sigma & circle & Elliptical \\
\hline$\sigma_{d}$ & $\frac{1}{2 \sqrt{3}}$ & $\frac{4}{\sqrt{b^{2}+2}} x$ \\
\hline$\sigma_{a}$ & $\frac{1}{4}$ & $\sqrt{\frac{b^{2}}{48}+\frac{\left(3 b^{4}-4 b^{2}+12\right)}{768}}$ \\
\hline$\sigma_{b a}$ & $\frac{1}{2 \sqrt{6}}$ & $\frac{b}{2 \sqrt{12}}$ \\
\hline$\sigma_{c}$ & $\frac{1}{2 \sqrt{2}}$ & $\begin{array}{c}\frac{9 \mathrm{~b}^{6}-2 \mathrm{~b}^{4}-4 \mathrm{~b}^{2}+72}{(96)^{2}}- \\
\frac{1}{36}\left(\frac{\mathrm{b}^{2}}{2}-1\right)^{2} \frac{\mathrm{b}^{2}}{\left(2 \mathrm{~b}^{2}+4\right)} \\
+\left(\frac{\left(\mathrm{b}^{2}+2\right)}{12}\right)^{2} \\
\sqrt{\left[\frac{1}{6}\left(\frac{\mathrm{b}^{2}}{2}-1\right) \frac{\left(\mathrm{b}^{2}+2\right)}{16}\left(\frac{\mathrm{b}^{2}-2}{\mathrm{~b}^{2}+2}\right)-\left(\frac{\mathrm{b}^{2}}{2}+1\right)\right]^{2}}\end{array}$ \\
\hline$\sigma_{b c}$ & $\frac{1}{6 \sqrt{2}}$ & $\frac{\sqrt{9 b^{6}-2 b^{4}-4 b^{2}+72}}{96}$ \\
\hline$\sigma_{s}$ & $\frac{2}{3 \sqrt{5}}$ & $\begin{aligned} \rho^{4}= & \sqrt{\frac{45 b^{8}-120 b^{6}+376 b^{4}-480 b^{2}+720}{184320}} E_{9} \\
& -\frac{1}{2} \frac{\sqrt{2} b^{2}}{2 \sqrt{9 b^{4}-12 b^{2}+36}} E_{6}\left(\frac{b^{2}}{2}-1\right) \\
& +\frac{1}{2} \frac{\sqrt{3 b^{4}-4 b^{2}+12}}{2 \sqrt{48}} E_{4}\left[\left(\frac{b^{2}}{2}+1\right)-\frac{9}{22}\left(\frac{b^{2}}{2}-1\right)\right]\end{aligned}$ \\
\hline$\sigma_{b s}$ & $\frac{1}{6 \sqrt{5}}$ & $\sqrt{\frac{45 b^{8}-120 b^{6}+376 b^{4}-480 b^{2}+720}{30720}}$ \\
\hline
\end{tabular}

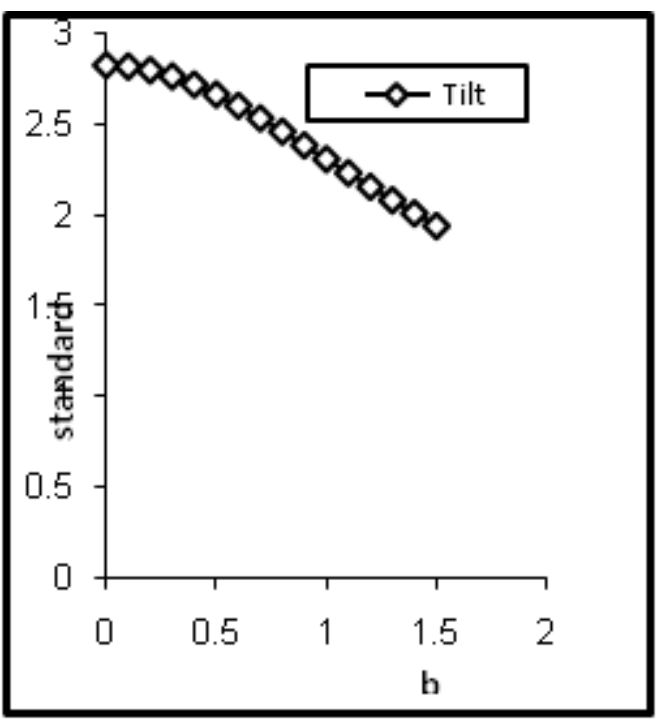

Volume 5 Issue 2, February 2016 


\section{International Journal of Science and Research (IJSR) \\ ISSN (Online): 2319-7064}

Index Copernicus Value (2013): 6.14 | Impact Factor (2014): 5.611

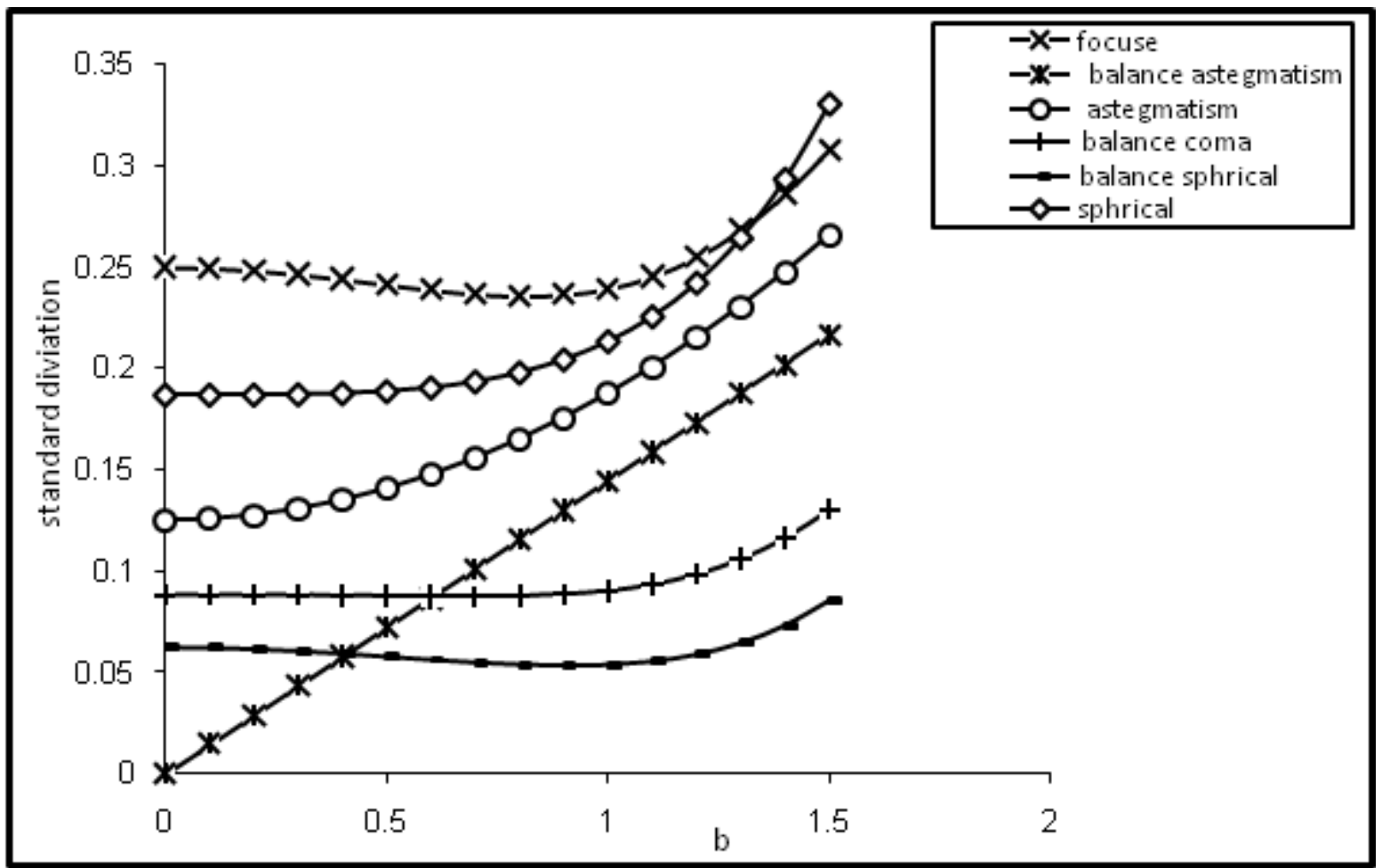

Figure 4: The standard deviation for balanced and un-balanced aberrations for focus, astigmatisim ,coma, and spherical as a function of $b$.

From above two figures, it's obvious that $\mathrm{s}$ of tilt error is decreased as aspect ratio $b$ decreased till it reach the value of spherical aperture when $b=\sqrt{2}$, while the rest are decreased with increasing $b$.

\section{Conclusions}

There are many conclusions reached in this research:

1. Zernike polynomials limits appropriate only for circularslot and are not appropriate for any other slot, because they lose orthogonal property. So it -was treated in a manner Cram - Schmidt orthogonality with the borders of the new slot to be orthogonal to get the property back on the borders of the new slot.

2. Zernike polynomials limits oval oblique angle $(\pi / 4)$ with the $\mathrm{x}$-axis has no axial symmetry as is the case of circular slot because it cannot be written in terms of one kind of trigonometric functions (cos or sin). Which, as is evident from the table (3-7), the border $1,2,4,5,6,9$, 12,13 and 16 can only be written in terms of a pocket or pocket only fully.

3. Zernike polynomials limits oval oblique angle $(\pi / 4)$ with the $\mathrm{x}$-axis be appropriate to describe the function of the slot aberration oval oblique.

4. This Almtadeddat be non-symmetrical circular, as is the case of circular aperture, and this is evident from the lack of written polar coordinates separately.

5. Zernike polynomials limits oval oblique angle $(\pi / 4)$ with the $\mathrm{x}$-axis return to the borders of Zernike polynomials circular when $b=\sqrt{2}$.

6. Squared values rate is equal to the total transactions Zernike boxes while covariance be equal to the total transactions Zernike boxes, except for the first term.

7. As in the Zernike polynomials limits circular, the rate of the value of the function of each aberration Zernike polynomials oval at an angle $(\pi / 4)$ with the $x$-axis is equal to zero, except for the first term, in other words, the rate of value be equal to Zernike first coefficient (or piston met).

8. Astigmatism aberration balance with defect length deviation and aberration caudatus balance with tilt and this resembles the corresponding limits in Zernike circular

9. In oval aperture oblique angle $(\pi / 4)$ with the $\mathrm{x}$-axis spherical aberration balance with focal length deviation and aberration Astigmatism, and this is different from the circular aperture in the balance as the spherical aberration with focal length aberration deviation only.

10. The standard deviation of aberrations depends on the bvalue and devolve these values to the values of the circular slot when $b=\sqrt{ } 2$.

\section{References}

[1] F. Zernike, "Diffraction theory of the knife-edge test and it improved form: the phase-contrast method," Physical 1,689-704 (1934).

[2] R. J. Noll "Zernike polynomials and atmospheric turbulence" J. Opt. Soc. Am., 66, 3 , (1976).

[3] R. webb "Zernike polynomial description of ophthalmic surfaces", Applied Optics , 3, 38, (1992)

[4] D. R. Iskander, M. J. Collins, and B. Davis, "Optimal modeling of corneal surfaces with Zernike polynomials", IEEE Trans. Biomed. Eng. 48, 1, 87-95, (2001).

[5] Y. P. Kathuria" Fresnel and far-field diffraction due to an elliptical aperture" J. Opt. Soc. Am., 2, 6, 852, (1985)

[6] J. A. Díaz and R. Navarro, " Orthonormal polynomials for elliptical Wavefrontswith an arbitrary orientation", App. Op., 53, 10, 2051, (2014).

\section{Volume 5 Issue 2, February 2016}




\section{International Journal of Science and Research (IJSR) \\ ISSN (Online): 2319-7064}

Index Copernicus Value (2013): 6.14 | Impact Factor (2014): 5.611

[7] V. N. Mahajan and G. m. Dai, "Orthonormal polynomials in wavefront analysis: analytical solution", J. Opt. Soc. Am. A ,24, 9 America,(2007).

[8] Sundus Y. Hasan and Ali S. Shaker "Study of Zernike polynomials of an elliptical aperture obscured with an elliptical obscuration," APPLIED OPTICS, 51, 35 (2012).

[9] Sundus Y. Hasan and Widad H. Tarkhan"Point Spread Function For Elliptical Aperture Inclined at $\pi / 4$ angle with x-axis" ISSN, 5,2, 2014

[10]Larry N. Thibos, Raymond A. Applegate, James Schwiegerling, and Robert Webb, "Standards for reporting the optical aberrations of eyes" Journal of Refractive Surgery, 18.5, 2002.

[11] Takuya Yokozawa, Daisuke Takahashi, TaisukeBoku, and Mitsuhisa Sato, "Efficient Parallel Implementation of Classical Gram-Schmidt Orthogonalization Using Matrix Multiplication",Proceedings of Fourth International Workshop on Parallel matrix Algorithms and Applications, 2006. 